\title{
Post Market Surveillance of VERTAUX Pedicle Screws System to Treat Spondylolisthesis Related Problems
}

\author{
Mohit Kumar $^{2 *}$, Pooja Rawat ${ }^{1}$ and Gaurav Luthra ${ }^{2}$ \\ ${ }^{1}$ Clinical Research Department, Auxein Medical Private Limited, Sonipat, Haryana, India \\ ${ }^{2}$ Regulatory Department, Auxein Medical Private Limited, Sonipat, Haryana, India \\ *Corresponding Author: Mohit Kumar, Regulatory Department, Auxein Medical Private Limited, Sonipat, Haryana, India.
}

Received: October 11, 2019; Published: October 23, 2019

DOI: $10.31080 /$ ASOR.2019.02.0118

\begin{abstract}
Background: Problems related to the Spondylolisthesis are very common now days due to the modern life style of the today's generation. The objective of this study was to study the postoperative performance of VERTAUX Pedicle Screws System manufactured using the titanium alloys.

Methods: This is a prospective study of 25 patients with one year follow up. The Spondylolisthesis related problems were treated with Spinal System- VERTAUX Pedicle Screws System manufactured by Auxein Medical Private Limited, India. X-ray was used to check the pre and post clinical conditions. Functional outcomes were assessed with Average VAS score.

Results: X-ray was showing good results. Before surgery Average VAS score was 7.44 and at the end of one year Average VAS score was 0.4 only. After 1 month, 3 months, 6 months and 9 months Average VAS score were calculated as 5.25, 4.18, 2.84 and 1.6 respectively. Conclusion: Treatment of Spondylolisthesis with VERTAUX Pedicle Screws System shows good outcomes with less complications. Keywords: Spondylolisthesis; Pedicle Screws System; Post Market Surveillance; Spinal Disorders
\end{abstract}

\section{Introduction}

Spondylolisthesis is the anterior or posterior displacement of a vertebra or the vertebral column in relation to the vertebrae. Spondylolisthesis is one of the major structural changes in the spine, which occasionally gives rise to back pain. Spondylolisthesis is displacement of a vertebra due to a defect in the pars [1]. Spondylolysis is a precipitating factor and can be classified as isthmic, dysplastic, degenerative, traumatic, and pathologic [2]. The lumbar spine is consists of 5 moveable vertebrae numbered L1- L5. The complex anatomy of the lumbar spine is a remarkable combination of these strong vertebrae, multiple bony elements linked by joint capsules, and flexible ligaments/tendons, large muscles, and highly sensitive nerves. It also has a complicated innervations and vascular supply.

Treatment of DS has long been a topic of debate, as multiple modalities are currently utilized. For example, in a recent analysis, 95,647 Medicare patients with a diagnosis of lumbar DS, 40\% were treated with corticosteroid injections, $37 \%$ were treated with physical therapy, and only $22 \%$ were treated surgically [3]. When considering surgical treatment variations with data from the Spine
Patient Outcomes Research Trial (SPORT), 7\% were treated with decompression alone, 21\% were treated with non-instrumented fusion, and $71 \%$ were treated with fusion surgery [4]. The reoperation rate is also high at $22 \%$ at 8 years after initial surgery. Nevertheless, surgical treatment of DS with symptomatic spinal stenosis has been shown to be cost-effective with gain of 0.43 quality adjusted life years (QALYs), or greater for patients with multilevel disease [4].

Pedicle Screws fixation with the rods and connectors is most commonly surgical procedure to treat Spondylolisthesis problems. The majority of these systems are made of stainless steel (Ss), but titanium-alloy (Ti-alloy) devices have recently been available on the market. Ti-alloy implants have several potential advantages over SS ones. High bioactivity and more flexibility may improve bone ingrowth and mechanical fixation, and the material also offers superior magnetic resonance imaging (MRI) and computed tomography (CT) resolution and significantly less signal interference. However, no data are available from loaded spinal constructs regarding bony ingrowth and mechanical fixation [5]. 


\section{Materials and Methods}

A Prospective study was conducted form May 2018 to April 2019 at Mesoamerican University, Quetzaltenango, Guatemala. A 25 patients between the age groups of 18-60 years with Isthmic or Degenerative spondylolisthesis were taken who did not get conservative management without significant relief of symptoms. Table for Inclusion and exclusion criteria are given below.

\begin{tabular}{|l|c|}
\hline \multicolumn{1}{|c|}{ Inclusion Criteria } & Exclusion Criteria \\
\hline $\begin{array}{l}\text { Patient between age group 18-60 } \\
\text { years with symptomatic spondylo- } \\
\text { listhesis not relieved by conservative } \\
\text { treatment. }\end{array}$ & $\begin{array}{c}\text { Patients with severe } \\
\text { spondylolisthesis grade } \\
\text { IV }\end{array}$ \\
\hline $\begin{array}{l}\text { Spondylolisthesis between grade I - } \\
\text { III }\end{array}$ & $\begin{array}{c}\text { Patients with failed } \\
\text { previous lumbar } \\
\text { surgery. }\end{array}$ \\
\hline $\begin{array}{l}\text { Isthmic or degenerative spondylolis- } \\
\text { thesis were only included. }\end{array}$ & $\begin{array}{c}\text { Acute traumatic spon- } \\
\text { dylolisthesis }\end{array}$ \\
\hline
\end{tabular}

Table 1

A total of 25 consecutive patients meeting the following criteria were included in this study: isthmic and Degenerative and no additional flexibility-modifying procedures were required on the spine. The mean age at the time of operation was 33 years (range 18 - 60 years). The indications for surgery were isthmic spondylolisthesis in Isthmic (lytic) 17 Cases (68\%) and degenerative spondylolisthesis in 8 cases (32\%) operated. Spinal System- VERTAUX Pedicle Screws System manufactured by Auxein Medical Private Limited, Sonipat, Haryana, INDIA) were used for posterior spinal stabilization. The Myerding grading system was measures the percentage of vertebral slip forward over the body beneath. Patient physical fitness was also observed through America Society of anaesthesiologist (ASA) Grade. Pain Scale record from the patient using Visual Analog Scale (VAS). Follow up of the patient were taken on 1 month, 3month, 6 month, 9 month then 12 Month.

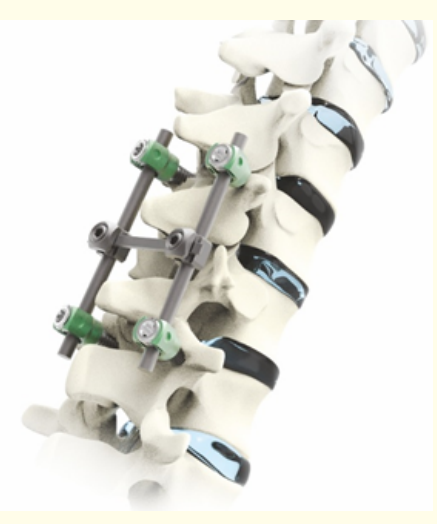

Figure 1: VERTAUX Pedicle Screws System.

\section{Results and Discussions}

According to ASA Grade all Paitent were felt in Grade 1 (Healthy individual with no Systemic Disease) Isthmic spondylolisthesis patients were more common than Degenerative, both being common in female patients and Grade 2 listhesis for 15 patients (60\%) was common then Grade 1 listhesis 7 patients (28\%) and Grade 3 for 3 patients (3\%). Physical therapy post-surgery for spondylolisthesis starts immediately. Ankle toe movements and deep breathing exercises started on the same day to prevent circulatory and respiratory complications. Post operatively the Intravenous Antibiotics were continued for 5 days followed by oral antibiotics for a period of 10 days. Regular wound check is done with the drain being removed on IInd post-operative day and the sutures being removed on average of 13 day. Brace is to be continued for 3 months postoperatively and patient removed it in average 3 months approx. Lumbar radiographs with anteroposterior, lateral and lateral standing flexion extension films and oblique view were obtained beginning with the 1st Month, 3rd month, 6th month, 9th month and 12-month (on 1 year) calculated From the day of surgery to check the stability. The fixation procedure is equally good achieving fusion and stability. The fusion status was judged, an evaluation done by an independent observer. The Average pain scale has been also calculated for the patients. Data is presented in figure 2 .

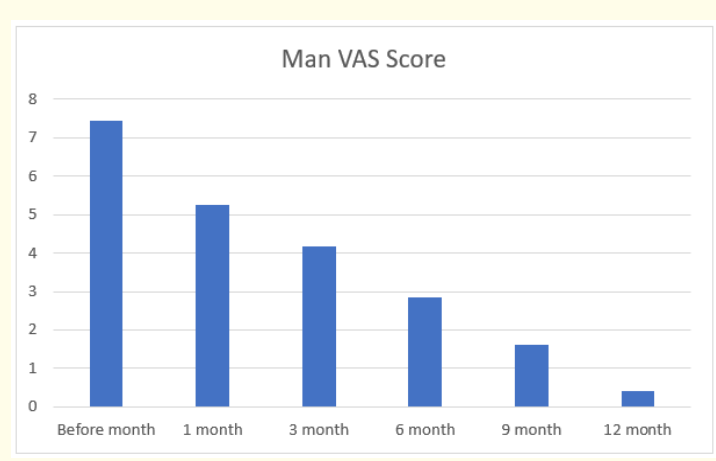

Figure 2: Mean VAS Score.

Before surgery Average VAS score was 7.44 and at the end of one year Average VAS score was 0.4 only. After 1 month, 3 months, 6 months and 9 months Average VAS score were calculated as 5.25, $4.18,2.84$ and 1.6 respectively. All the patients included in the study showed satisfactory bony fusion as judged by solid fusion at both facets joint. Radiological fusion was seen earliest on 3rd month check x- ray. Pre and post operation lateral radiograph of L-S spine showing forward displacement of L5 over S1 are given in the below figure 3 .

Dai., et al. studied that pedicle screws can reduce pain and improve spinal dysfunction in osteoporotic patients undergoing 


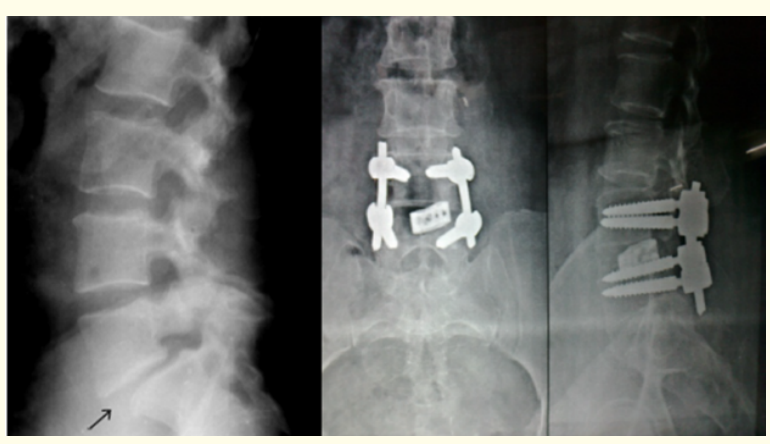

Figure 3: Pre and Post Operation X Rays.

osteoporotic spine surgery [6]. Cook., et al. also studied the mechanical and clinical performance of the pedicle screws fixation system they also not find any complication in the treatment with the pedicle screw system [7].

The present study demonstrates use of VERTAUX Pedicle Screws System for Spondylolisthesis with successful outcome. With a follow-up of 1 years no complications were reported. Joveniaux JK., et al. conducted a follow-up of 24 months and they concluded that pedicle screws system is safer to use [8]. In our study there were no complications noticed related to spinal biomechanics in study and Hardware related complications were not encountered in this so with complete success rate there was no report of reoperation thereby achieving good functional results with excellent morbidity of the muscles. The experience of using of VERTAUX Pedicle Screws System was significant to be used for locking plate system for problems related to the spondylolisthesis.

\section{Bibliography}

1. McGregor AH., et al. "The use of interventional open MRI to assess the kinematics of the lumbar spine in patients with spondylolisthesis". Spine 27 (2002): 1582-1586.

2. Herman MJ and Pizzutillo PD. "Spondylolysis and spondylolisthesis in the child and adolescent: a new classification". Clinical Orthopaedics and Related Research 434 (2005): 46-54.

3. Sclafani JA., et al. "Descriptive analysis of spinal neuroaxial injections, surgical interventions, and physical therapy utilization for degenerative lumbar spondylolisthesis within medicare beneficiaries from 2000 to 2011". Spine 42.4 (2017): 240-246.

4. Gerling MC., et al. "Risks factors for reoperation in patients treated surgically for degenerative spondylolisthesis: a sub analysis of the 8 year data from the SPORT trial". Spine 42.20 (2017): 1559-1569.
5. Christensen FB., et al. "Titanium-alloy enhances bone-pedicle screw fixation: mechanical and histomorphometrical results of titanium-alloy versus stainless steel". European Spine Journal 9.2 (2000): 97-103.

6. Dai., et al. "Surgical treatment of the osteoporotic spine with bone cement-injectable cannulated pedicle screw fixation: technical description and preliminary application in 43 patients". Clinics 70.2 (2015): 114-119.

7. Cook SD., et al. "Biomechanical evaluation and preliminary clinical experience with an expansive pedicle screw design". Journal of Spinal Disorders and Techniques 13.3 (2000): 230236.

8. Houten JK., et al. "Clinical assessment of percutaneous lumbar pedicle screw placement using the 0-arm multidimensional surgical imaging system". Neurosurgery 70.4 (2012): 990-995.

Volume 2 Issue 11 November 2019 (C) All rights are reserved by Mohit Kumar., et al. 